\title{
Ethical dilemmas in pandemics - lessons from COVID -19
}

\author{
Mohamed Rifdy Mohideen', Felicity Mishan Ng Yiwey ${ }^{2}$ \\ ${ }^{\prime}$ Department of Internal Medicine, International Medical University, IMU Campus, 6 Jalan Indah, Taman Sri Kenangan , \\ 83000, Batu Pahat, Malaysia. \\ ${ }^{2}$ International Medical University, Bukit Jalil, Kuala Lumpur, Malaysia.
}

Correspondence: Professor Mohamed Rifdy Mohideen

e-mail: mohamed_rifdy@imu.edu.my

(D) https://orcid.org/0000-0002-9777-8067

Keywords: COVID-19, ethics, healthcare worker, pandemic, public health, research

\section{Introduction}

At the end of December 2019, the Chinese government sent out a public alert that a "pneumonia of unknown cause" had been identified in the city of Wuhan. Soon after, the causative organism was identified to be a novel coronavirus and named SARS-CoV-2 as the infection, COVID-19 (1). Since then, the infection has rapidly spread throughout China and reached multiple continents over a few weeks and was officially declared a pandemic by the World Health Organisation (2). The outbreak of COVID-19 has imposed a substantial health burden globally creating an international public health emergency.

With the world economy in a tailspin, financial market indices cratering, and international travel and unfettered movement of humans and goods severely disrupted, many nations have been exposed for a lack of preparedness for a global pandemic of this magnitude. This crisis has tested emergency services in every country, leaving bare the shortcomings of health systems including those in the wealthy industrialised nations. Compounding this is a devastating impact on the livelihood of vulnerable sections of society that has left governments scrambling for bailout packages for their people. Adding to it is fear and helplessness in people on a scale not seen previously except during natural disasters and genocidal wars.

The COVID-19, in comparison with other coronavirus infections like SARS (Severe Acute
Respiratory Syndrome) and MERS (Middle East Respiratory Syndrome), is a more contagious but a less deadly disease (3). Risk is enhanced for the elderly and those with serious underlying medical conditions, although the young and the previously healthy are not totally immune. Spread is mainly through respiratory droplets while other modes of transmission have been reported (4).

The transmissibility depends on the biological properties of the coronavirus and contact patterns which may reduce with public health intervention. There are reports that spread may occur during the incubation period before symptoms emerge, justifying some early aggressive preventive measures (5).

The tried and tested public health measures that have been implemented during infectious outbreaks of this nature are isolation, quarantine and social distancing. These measures aim at limiting social interaction and physical distancing to prevent the infection from spreading further. The support for such public health interventions are not uniform; some consider this insufficient while others oppose it severely as it restricts free movement.

When such threats emerge, a wide range of legal avenues are exercised under public health laws and emergency statutes. Closure or restricted use of public spaces, shuttering of business premises, restricting hours of trade, discontinuation of work, enforcing cordon sanitaire, curfews, price control and travel restrictions are some of the available legal powers. 
This paper examines the ethical challenges and dilemmas posed by public health actions, research initiatives and healthcare worker vulnerability. Additionally, we will explore how these dilemmas can be managed in a way that respects ethical norms, constitutional safeguards and democratic values.

\section{Public health actions}

Public health is critically about population health, protecting or improving the health of everyone in a society or entire population with actions for the greatest good for the greatest number. It is the first and the quickest mode of action that can be deployed to interrupt transmission chains during infectious outbreaks: isolating the infected and treating the sickest and distancing the others from each other.

Public health actions such as vaccination in nonurgent situations have generally taken place through voluntary cooperation of individuals or community than on compulsion. Many measures that are taken are without dispute and are deemed necessary and uncontroversial such as isolation of a confirmed case in a hospital or healthcare facility with adequate safeguards for further spread of the infection.

During the efforts to contain the spread of the infection effectively, the values that the society holds dear should not be a casualty and existing injustices should not worsen. Values imbue all areas and levels of public health theory and practice and are culturally bound. The key Siracusa Principles, adopted by the UN Economic and Social Council in 1984 recommends that restrictions should, at a minimum, be among others be least intrusive and restrictive available to reach the objective, be based on scientific evidence and neither arbitrary nor discriminatory in application of limited duration, respectful of human dignity, and subject to review (6).

In these crisis situations, it is inevitable that individual human rights and civil liberties will be restricted for the greater good of the public. However, the limits placed on individual rights should be essential, proportionate, nondiscriminatory, just and in full compliance with national and international laws. The local laws and cultural context will nevertheless influence some of these actions. Vulnerable groups need to be protected during these times: the disabled, elderly, migrants, asylum seekers, homeless and prisoners.

\section{Sanctity of life}

A pandemic is a time when the sanctity of human life is tested, as resources dwindle, and brutal utilitarian ideas prevail. With every passing day, state powers expand, authorities overreach, and individual rights squeezed in a frantic effort to contain the spread. It is understandable that governments blinded by many unknown factors need flexibility to act decisively. However, the society expects it to act for the common good with fairness and proportionately.

Individual human rights of liberty and privacy are a luxury and often a casualty in times of such exigencies. Reports from different parts of the world indicate several human rights violations including freedom of expression, censorship, discrimination, arbitrary detention, and xenophobia during this pandemic (7).

\section{Surveillance}

Public health surveillance is a crucial arm of a suite of countermeasures that are employed in the early part of an infectious outbreak. The data that is gathered provides the scientific basis to understand the transmission of the infection and provide early warnings to plan a targeted response.

Legally, public health law may allow for the collection of sensitive information related to personal health, travel and contacts particularly at the beginning of outbreaks (8). When these data are collected in the interest of protecting a broader public, people cannot expect the same level of personal health privacy that is operative during clinical encounters. However, it is expected that authorities keep the information confidential and process it anonymously and limit it to the period of the crisis. A breach of this right may cause social and psychological harm. Disclosing information publicly that allows indirect identification of individuals or sharing it with others not entitled to have it, are serious breaches of privacy.

There is justification to forego informed consent procedures when it can be shown that the collected data is useful for accurate portrayal of spread and 
beneficial to protect the vulnerable population (9). Legitimate authorities should be involved in the collection of data and it should be limited to the infection under study.

Governments around the world are using location information to track citizens to identify travel patterns and alerting users to locations where infection has been detected. Cell phone carriers in several European countries shared customers' locations with their respective governments to track the spread of the virus and success of containment methods (10). Some countries required citizens to install software on their smart phones which predicts people's health status, tracked their location and movements while others had their data in the public domain where people with COVID-19 had previously visited (11).

The extensive technological use is novel in pandemics, beneficial in the battle for containment of the spread of the infection but brings out new challenges. Under the public health emergency, authorities seeking such information in many countries may not need any judicial permission. The concern is about the abuse of these digital forays when the pandemic is over, as governments will be reluctant to dismantle these intrusive surveillance methods and continue well into the postpandemic phase. The aggregated and anonymised data that is available should be less granular with additional measures put in place to protect the personal data of infected and those at risk.

\section{Quarantine, isolation and social distancing}

Isolation and quarantine are the two public health actions that illustrate the most extreme of state powers: the power to deprive the individual of free movement and existing liberties.

Dating back to as early as the fourteenth century, quarantine has been used as an important strategy in the containment of contagious diseases (12). It is described as means to restrict the movement and activities of asymptomatic people who have had exposure to an infectious disease, usually for the incubation period of the suspected pathogen. Quarantine aims to achieve two important goals - to stop the disease transmission within a community and to help with identifying individuals under surveillance for medical care.
Social or physical distancing, in contrast to quarantine measures is aimed at limiting the physical interactions between individuals of a community. This involves increasing the physical space between individuals by ensuring a safe distance of at least six feet. Both measures aim to limit disease transmission as common goals (13).

Social or physical distancing is highly disruptive to daily life and felt heavily by the vulnerable and economically disadvantaged sections of society. With risk of contracting the infection disproportionate, a low-risk healthy young person rides it out by staying at home at great personal cost while the benefits accrue to the most vulnerable sections of society. Chronic social isolation is not without its adverse effects and when prolonged can increase the risk of a number of health problems including death (14). Closures of schools, universities and workplaces push students, employees and parents to stay and work from home. If implemented fairly and shown to be effective to slow the transmission through scientific studies as shown in the current COVID-19 outbreak in China, public adherence to the measures are more likely (15).

In some countries, reports are emerging of coercive and at times heavy handed methods enforced by security authorities to control the population and groups. When the population appears to be untrustworthy of following quarantining or physical distancing measures, curfews have been imposed sometimes at short notice. Such arbitrary enforcement violates the rule of least infringement of human rights.

An ideal solution for nations as part of epidemic preparedness, draft and follow proper quarantine policies in a transparent manner with wide consultation. When done with adequate planning, not only will the standards for ethical principles be met but there would be a higher likelihood of public acceptance for such a policy.

\section{Stigmatisation and shaming}

Stigmatisation is an unfortunate consequence of infectious diseases. In the past, tuberculosis, syphilis and more recently HIV infection were notorious examples. Shaming is another weapon used for 
rule-breakers but offers a prospect of reintegration to society that stigmatization does not (16). Some justify these strategies in chronic negative behaviours such as smoking to dissuade from such practices (16). Others view it as paternalism and an affront to the liberties and rights of the individual. It would indeed be an uneasy act to include in the repertoire of public health measures regardless of whether the goal was to protect people from harming others or themselves.

These unfortunate practices are further compounded when the media picks up the story and the location of individuals is divulged leaving the person to be publicly identified, criticised and humiliated. In some instances, names and photographs have been posted in newspapers and websites similar to the pursuit of a suspected criminal.

\section{Healthcare worker hazards and rights}

With their avowed commitment to care for the sick and injured, doctors and other healthcare workers have an obligation to provide urgent medical care during health emergencies. The standard risks are implicit in the nature of the job and in the ethical practice of medicine. There is significant risk of infecting themselves and of death as seen among healthcare workers during the Ebola, SARS, MERS and in the current COVID-19 pandemic $(17,18)$.

It is reasonable to expect, that the frontline health staff receive sufficient protection from being exposed to hazards that put them at risk of infection. Reports of insufficient and inadequate personal protective equipment (PPE) to frontline health workers during patient encounters with confirmed or suspected cases have raised concerns during this crisis with conflicting and confusing guidelines issued by authorities about when these protective measures should be used (19). With supplies dwindling in many countries, these scarce resources have been limited to high-risk procedures and forced to reuse single-use items of PPE have also raised alarms among the healthcare workers.

An obligation of the state and health care institutions exists to safeguard the rights and safety of healthcare workers as they are irreplaceable. It begins with good planning of adequate medical supplies and staffing to ensure a safe work environment.

\section{Resource allocation}

Other contentious areas in managing COVID-19 patients involve identifying patients for ventilation and resuscitation. When a hospital does not have enough staff or equipment to provide ventilatory support, priority is assigned to patients with the greatest likelihood of benefit from it. The ethical rationale for such an approach is a strictly utilitarian one. Excluding certain patient categories such as those with severe lung disease or cognitive impairment may appear reasonable but ethically problematic. A fair and consistent way to deal in such situations as practised in some US hospitals is to use a score-based framework (using multiple criteria) which includes all morally relevant values into an objective decision-making process (20).

At times of crisis, as seen in this pandemic and in disaster situations, hospitals would relocate its usual facilities, staff and equipment to prioritise emergent needs. As a result, elective surgery and nonurgent investigations will be deferred, staff reassigned to other units and supplies deployed to where it is needed most. When these are done ad hoc without previous planning or clear communication it usually leads to tensions and discontent among the stakeholders.

The dilemmas that occur on the ground need to be resolved invoking all the principles of ethics including beneficence/non-maleficence, professional integrity, utility and justice to balance the benefits to the patient and harm to the caring team.

These difficult decisions taken under unusual demands on resources affecting the sanctity of life leads to moral injury to health workers. In the aftermath, as observed in other epidemics, burnout, psychological stress and anxiety is likely to be present among the healthcare $\operatorname{staff}(21)$.

\section{Duty and law}

In carrying out duties, the healthcare worker needs to comply with the law of the land. Countries and states may invoke additional powers beyond those in the regular public health laws and promulgate new ones to deal with the imminent threat. Matters of notification, disposal of dead bodies are examples of inflexible duties as it allows little or no discretion 
in their application (8). This puts the worker in moral conflict with respect to non-discrimination, and human rights. Such disputes should be solved through community engagement and mediation. When laws governing public health matters are reviewed in the future, the idea that much of the work of public health action is about risk assessment should be recognised (8).

\section{Public health emergency research}

The key to proper public health policy in infections is knowing the science behind transmission. Research through innovative therapies and vaccine technology will ultimately provide lasting solutions to combat this viral infection.

The need to conduct research during epidemics such as in COVID-19 is undisputed and urgent. Some see it as an ethical obligation to conduct research during the epidemic while others are concerned that it may draw efforts away from providing clinical care. In the absence of an effective therapeutic agent for COVID-19 infection, the immediate need is to embark on safe and effective interventions.

Significant ethical issues emerge when untested therapies are used without its clinical effectiveness established in clinical trials. In desperation, drugs used in similar viral infections and other conditions are repurposed based on experimental evidence at best. Hydroxychloroquine is one such example for its easy availability and low cost.

Undoubtedly, randomized controlled clinical trials sit on the pinnacle of the level of evidence when it comes to establishing the effectiveness of a treatment, device, or other intervention. Although flexibility of clinical trial designs is justifiable due to the urgency, the scientific validity and ethical integrity should not be sacrificed in pursuit of new therapeutic modalities.

Valuable lessons have been learnt in conducting research during the most recent Ebola outbreak in West Africa in late 2013 despite the therapeutic trials failing to achieve efficacy endpoints. The expert committee in charge of the research identified seven moral requirements that should guide all clinical research including research conducted during epidemics: scientific and social value, respect for persons, community engagement, concern for participant welfare and interests, a favourable risk benefit balance, justice in the distribution of benefits and burdens, and post-trial access (22).

Providing protective immunity through vaccination is undoubtedly the most effective strategy to prevent and control this infection in the long term. At the best of times, human vaccine development involves careful study design, lengthy and stepped sequence of testing and ethical oversight. Prior to an outbreak, there is no way of knowing the strain of the organism for conducting vaccine studies beforehand. The dilemma is further compounded due to strict adherence to international guidelines on human clinical trials.

In the rush to test an experimental vaccine, new approaches are adopted where the traditional steps of prior animal testing are abandoned before human experimentation. Human challenge trials, which sidestep early safety testing (Phase 3 ) of standard trials, is proposed to cut waiting time for vaccine efficacy (23). Significant ethical issues are raised as no effective treatment is yet available for any volunteers who may develop severe infection from the live virus. Limiting the risk by recruiting young healthy volunteers, providing excellent aftercare and close monitoring of subjects in high level medical facilities minimises the risk but does not eliminate the occurrence of unforeseen complications. Although these approaches gain valuable time, it is unlikely an effective vaccine for coronavirus will be available during this epidemic.

A robust peer-review of the research proposal can be undertaken to offset some of these constraints. Another approach is to adopt a "core protocol," model, which would allow a single clinical trial to extend across multiple infectious disease outbreaks (24). This approach is to avoid publishing data from studies that have not reached their pre-specified outcomes due to waning of the epidemic. The data would be stored, rather than reported, and the trial would continue the next time another outbreak appears.

\section{Conclusions}

Global health realities have exposed ethical transgressions, social inequalities and healthcare vulnerabilities by this contagion. In a globalized 
world, the threats from novel pathogens crossing national boundaries require a new world order of cooperation and solidarity of international health, sadly missing at the start of this crisis. Preparedness for emerging diseases should include rigorous consideration of ethical issues in advance of future outbreaks to maintain public trust and safeguard the population health.

The crises should not be used to erode civil liberties. Restrictions of rights and freedom should have a legal basis and not be arbitrary or discriminatory. Exploring less intrusive evidence-based alternatives will make public health actions less controversial and more acceptable. Good public health that respects civil liberties will enhance public health action. Hopefully, lessons learnt from this pandemic will create a renewed urgency for better preparedness.

\section{Acknowledgement}

We are grateful to Prof. Velayudhan Menon for reviewing the manuscript and for his useful suggestions.

\section{References}

1. Zhu N, Zhang D, Wang W, Li X, Yang B, et al. A novel coronavirus from patients with pneumonia in China, 2019. NEnglJMed, 2020; 382(8): 727-733.

2. World Health Organization. WHO Director-General's opening remarks at the media briefing on COVID-19-11 March 2020: Available at: https://www.who.int/dg/ speeches/detail/who-director-general-s-opening-remarksat-the-media-briefing-on-covid-19---11-March-2020. [Accessed 18 April 2020].

3. Huang C, Wang Y, Li X, Ren L, Zhao J, et al. Clinical features of patients infected with 2019 novel coronavirus in Wuhan, China. The Lancet, 2020;395(10223): 497-506.

4. van Doremalen N, Bushmaker T, Morris DH, Holbrook MG, Gamble A, et al. Aerosol and surface stability of SARS-CoV-2 as compared with SARS-CoV-1. $N$ Engl $J$ Med, 2020; 382: 1564-1567.

5. Mizumoto K, Kagaya K, Zarebski A, Chowell G. Estimating the asymptomatic proportion of coronavirus disease 2019 (COVID-19) cases on board the Diamond Princess cruise ship, Yokohama, Japan 2020. Euro Surveill,
2020; 25(10): 2000180. DOI: $10.2807 / 1560$ 7917.ES.2020.25.10.2000180.

6. UN Commission on Human Rights, The Siracusa Principles on the Limitation and Derogation Provisions in the International Covenant on Civil and Political Rights, 28 September1984, E/CN.4/1985/4: Available at: https://www.refworld.org/docid/4672bc122.html [Accessed 18 April 2020]

7. Seven ways the coronavirus affects human rights: Available at: https://www.amnesty.org/en/latest/news/ 2020/02/explainer-seven-ways-the-coronavirus-affectshuman-rights/ [accessed 18 April 2020]

8. Martin R. The limits of law in the protection of public health and the role of public health ethics. Public Health. 2006; 120(Suppl): 71-80.

DOI: $10.1016 /$ j.puhe.2006.07.0

9. Klinger C, Silva DS, Schuermann C, Reis AA, Saxena A, et $a l$. Ethical issues in public health surveillance: a systematic qualitative review. BMC Public Health, 2017; 17: 295. DOI: $10.1186 / \mathrm{s} 12889-017-4200-4$.

10. Countries track mobile location to fight COVID-19: Accessed at: https://www.gpsworld.com/19-countriestrack-mobile-locations-to-fight-covid-19/: [Accessed 20 April 2020]

11. Countries are using apps and data networks to keep tabs on the pandemic: Accessed at: https://www.economist.com/ briefing/2020/03/26/countries-are-using-apps-and-datanetworks-to-keep-tabs-on-the-pandemic [Accessed 20 April 2020].

12. Tognotti E. Lessons from the history of quarantine, from plague to influenza A. Emerg Infect Dis, 2013; 19(2): 254-259.

13. Coronavirus, Social and Physical Distancing and SelfQuarantine: Accessed at: https://www.hopkinsmedicine. org/health/conditions-and-diseases/coronavirus/ coronavirus-social-distancing-and-self-quarantine [Accessed 20 April 2020]

14. Holt-Lunstad J, Smith TB, Layton JB. Social relationships and mortality risk: a meta-analytic review. PLoS Med, 2010 Jul 27; 7(7): e1000316.

DOI: 10.1371/journal.pmed.1000316.

15. Maier BF, Brockmann D. Effective containment explains subexponential growth in recent confirmed COVID-19 cases in China. Science, 2020 May 15; 368(6492): 742-46. 
16. Bayer R. Stigma and the ethics of public health: not can we but should we. Soc Sci Med. 2008; 67(3): 463-472.

17. Liu S, Chan T-C, Chu Y-T, Wu JT-S, Geng X, et al. Comparative epidemiology of human infections with Middle East Respiratory Syndrome and Severe Acute Respiratory Syndrome Coronaviruses among healthcare personnel. PLoS ONE, 11(3): e0149988. DOI: 10.1371/journal. pone. 0149988 .

18. At least 130 US health care workers dead from COVID-19: Accessed at: https://www.wsws.org/en/articles/2020 /04/25/heal-a25.html [Accessed 5-May-2020].

19. Public Health England Guidance. COVID-19 personal protective equipment (PPE) Updated 17 April 2020: Accessed at: https://www.gov.uk/government/ publications/wuhan-novel-coronavirus-infectionprevention-and-control/covid-19-personal-protectiveequipment-ppe [Accessed 21-April-2020].

20. White DB, Katz MH, Luce JM Lo B. Who should receive life support during a public health emergency? Using ethical principles to improve allocation decisions. Ann Intern Med, 2009; 150: 132-138.
21. Lee SM, Kang WS, Cho AR, Kim T, Park JK. Psychological impact of the 2015 MERS outbreak on hospital workers and quarantined hemodialysis patients. Compr Psychiatry, 2018; 87: 123-127.

22. National Academies of Sciences, Engineering, and Medicine 2017. Integrating Clinical Research into Epidemic Response: The Ebola Experience. Washington, DC: The National Academies Press. https://doi.org/ $10.17226 / 24739$.

23. Plotkin SA, Caplan A. Extraordinary diseases require extraordinary solutions. Vaccine, 2020; 38(24): 39873988.

24. Dean NE, Gsell PS, Brookmeyer R, Crawford FW, Donnelly CA, et al. Creating a framework for conducting randomized clinical trials during disease outbreaks. NEngl JMed, 2020; 382(14): 1366-1369. 